\title{
FIRST STUDY OF THE ECOLOGICAL STATUS IN THE ATLANTIC COAST OF MOROCCO USING THE BROWN SEAWEED CYSTOSEIRA TAMARISCIFOLIA
}

\author{
BOUNDIR, Y..$^{1,2,3^{*}}$ - HASNI, M. ${ }^{4,5}$ - RAFIK, F. ${ }^{2}-$ SABRI, H. ${ }^{1,3}$ - BAHAMMOU, N. $.^{1,3}-$ \\ CHEGgOUR, M. ${ }^{5}-$ ACHTAK, H. $^{2}-$ CHERIFI, O. ${ }^{1,3}$ \\ ${ }^{1}$ Laboratory of Hydrobiology, Ecotoxicology, Sanitation and Global Changes (LHEAC- \\ URAC33), Faculty of Sciences Semlalia, Cadi Ayyad University \\ Bd. Prince My Abdellah, PO Box 2390, 40000 Marrakesh, Morocco \\ ${ }^{2}$ Environment and Health Team, Polydisciplinary Faculty, Cadi Ayyad University \\ Route Sidi Bouzid PO Box 4162 Avenue Mohamed Belkhadir, 46000 Safi, Morocco \\ ${ }^{3}$ National Center for Studies and Research on Water and Energy (CNEREE), Cadi Ayyad \\ University \\ Avenue Abdelkrim Khattabi, PO Box 511, 40000 Marrakesh, Morocco \\ ${ }^{4}$ Faculty of Sciences, Ibn Zohr University, PO Box 8106, 80000 Agadir, Morocco \\ ${ }^{5}$ Biology department, Ecology unit, Ecole Normale Supérieure, Cadi Ayyad University \\ Hay Hassani Route d'Essaouira, PO Box 2400, 40000 Marrakesh, Morocco \\ ${ }^{*}$ Corresponding author \\ e-mail: younes.boundir@ced.uca.ma; phone: +21-263-884-6052; fax: +21-205-433-170 \\ (Received $5^{\text {th }}$ Jun 2019; accepted $25^{\text {th }}$ Oct 2019)
}

\begin{abstract}
This study is a first attempt to evaluate the toxic effects of prominent aquatic pollutants (nutrients and toxic metals) on the brown seaweed Bushy Rainbow Wrack (Cystoseira tamariscifolia) physiology along the Atlantic coast of Morocco. The physicochemistry (nutrients) of seawater, toxic metals (Chromium, Lead, Copper and Cadmium) and physiological parameters (Chlorophyll contents, Proline, Glycinebetaine and Total Phenolic Compounds) of the brown macroalgae Bushy Rainbow Wrack were studied in order to assess the pollution degree of 8 coastal areas. The results show that the toxic metal contents of Bushy Rainbow Wrack (especially Cadmium) and the concentration of phosphorus are correlated with stress physiological parameters, and inversely correlated with pigment contents. It shows that while these brown algae exist in the less polluted areas, their physiology is significantly affected. However, in the highly polluted areas, this brown seaweed disappears. Thus, this specie could be used for monitoring the pollution degree in coastal areas.
\end{abstract}

Keywords: environmental pollution, heavy metals, physicochemical parameters, algal physiology

\section{Introduction}

Cystoseira is a genus of brown macroalgae. Most of the species of this genus are very sensitive to pollution and to other anthropogenic pressures, and their numbers have diminished considerably during the last decades (Cormaci et al., 1999; Thibaut et al., 2005). With the rapid industrialization and economic development of coastal regions, heavy metals continue to be introduced into coastal zones, our country's coast must be in such a state that local organisms can live, develop and reproduce without hindrance. To achieve this goal, it is essential that pollutant inputs to water be reduced or even avoided, regardless of their origin: domestic, artisanal, industrial, agricultural or other sources (Blinda et al., 2013). Seaweeds are widely recognized as autogenic "ecosystem 
engineers" (Jones et al., 1994) or "foundation species" (Dayton, 1975). In general, the direct causes of change in marine biodiversity loss and coastal ecosystems, are: pollution, habitat destruction, increases in sedimentation, overexploitation of resources, climate change, and invasive species (Walker and Kendrick, 1998; Claudet and Fraschetti, 2010; Munday et al., 2013). The aim of this study is to portray the decrease of Bushy Rainbow Wrack (Cystoseira tamariscifolia (Hudson) Papenfuss) and to clarify the source and the conceivable reasons of its decrease in the Atlantic coast of Morocco.

\section{Materials and Methods}

\section{Location}

Eight (8) stations located on rocky substrates along the Atlantic coast of Morocco were chosen:

- Eljadida city with 2 stations: Sidi Bouzid coast: $33^{\circ} 13^{\prime} \mathrm{N}-8^{\circ} 55^{\prime} \mathrm{W}$ as a control area (S1). According to Moroccan beaches position, this station is ranked each year among the beaches called "Blue Flag". The Mohammed VI Foundation for the Protection of the Environment awards this distinction each year to beaches that fit the international standard norms of cleanliness (F.M.6, 2018). Jorf Lasfar: $33^{\circ} 07^{\prime} \mathrm{N}-8^{\circ} 37^{\prime} \mathrm{W}(\mathrm{S} 2)$ known as a polluted one. This area is characterized by the presence of multiple industrial units including a phosphate complex and a power thermal plant (Essedaoui et al., 2001; Kaimoussi et al., 2001; Ferssiwi et al., 2004).

- Safi city with 3 stations: Beddouza: $32^{\circ} 54^{\prime} \mathrm{N}-9^{\circ} 27^{\prime} \mathrm{W}$ (S3), less polluted station located $34 \mathrm{~km}$ from the industrial city of Safi (Goumri et al., 2018); Industrial Area: $32^{\circ} 28^{\prime} \mathrm{N}-9^{\circ} 24^{\prime} \mathrm{W}$ (S4) and Phosphate Area: $32^{\circ} 18^{\prime} \mathrm{N}-9^{\circ} 26^{\prime} \mathrm{W}$ (S5).

- Essaouira city where 3 stations were selected: Moulay Bouzerktoun $\left(31^{\circ} 63^{\prime} \mathrm{N}-9^{\circ} 67^{\prime} \mathrm{W}\right)(\mathrm{S} 6)$; located approximately $15 \mathrm{~km}$ from the enclosure of the city, it is less affected by anthropogenic activities, just an ephemeral tourism activity during the summer. Bab Doukala: $31^{\circ} 51^{\prime} \mathrm{N}-9^{\circ} 76^{\prime} \mathrm{W}$ (S7) and the port: $31^{\circ} 51^{\prime} \mathrm{N}-9^{\circ} 77^{\prime} \mathrm{W}(\mathrm{S} 8)$, receive domestic and some industrial releases (Sabri et al., 2017; Cherifi et al., 2018). Among them, 3 stations less polluted and 5 more polluted near to industrial factories (Fig. 1).

\section{Sampling}

Algal Samples were collected during Autumn 2017 to Summer 2018 at 0-5 meter depth depending on the geomorphology of the stations (Fig. 2) and following the principle of the quadrats. Then, washed in seawater, put in plastic packs and transported to the research center in a cooler for metal analysis. The samples expected for identification are preserved into alcohol at $10 \%$ while samples for physiology analysis are kept dry. Those of ocean water were collected during the same period, placed in a cooler and were conveyed fresh to the research center.

\section{Physicochemical parameters}

Concentration of inorganic nutrients of seawater (Phosphorus and Nitrogenous compounds) was measured according to AFNOR norms: T90-012 for Nitrate, T90-015 for Ammonium, T90-061 for Total nitrogen, T90-022 for Orthophosphate and T90-023 for Total phosphorus. Metal Concentrations of $\mathrm{Cd}, \mathrm{Cu}, \mathrm{Pb}$ and $\mathrm{Cr}$ were measured in seawater and the dried Bushy Rainbow Wrack according to Blinda et al (2013) and 
Topcuoglu et al (2003) methods, respectively. The samples were digested with concentrated nitric acid and analyzed using Atomic Absorption Spectrophotometer.

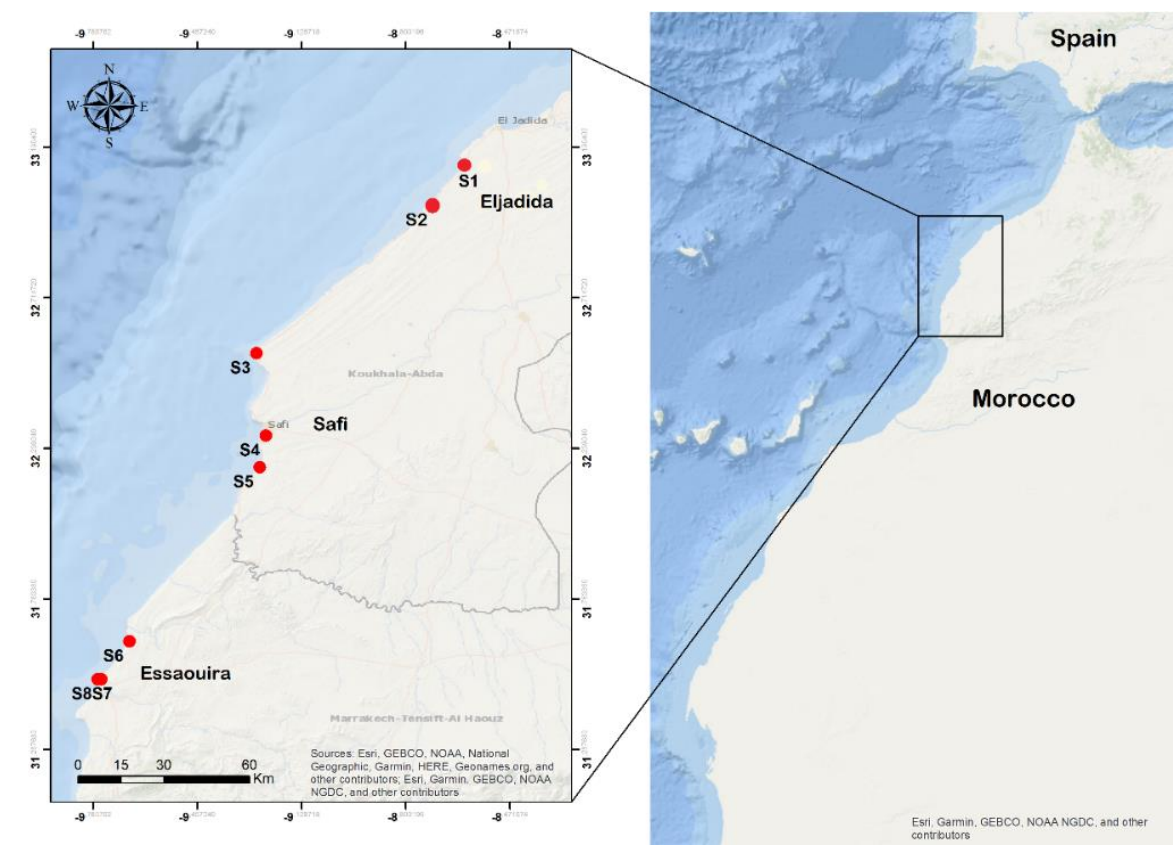

Figure 1. The location of the sampling points using ArcGIS, version 10.7. (S1) Sidi Bouzid:

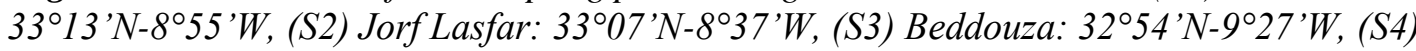

Industrial Area: $32^{\circ} 28^{\prime} \mathrm{N}-9^{\circ} 24^{\prime} \mathrm{W}$, (S5) Phosphate Area: $32^{\circ} 18^{\prime} \mathrm{N}-9^{\circ} 26^{\prime} \mathrm{W}$, (S6) Moulay

Bouzerktoun: $31^{\circ} 63^{\prime} \mathrm{N}-9^{\circ} 67^{\prime} \mathrm{W}$, (S7) Bab Doukala: 31 $51^{\circ} \mathrm{N}-9^{\circ} 76^{\prime} \mathrm{W}$, (S8) The port: $31^{\circ} 51^{\prime} \mathrm{N}-9^{\circ} 77^{\prime} \mathrm{W}$

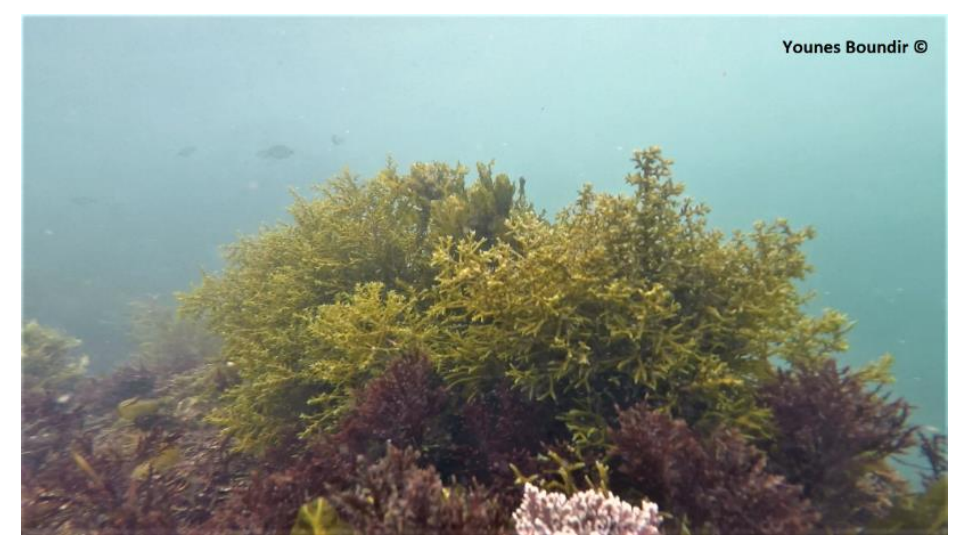

Figure 2. Bushy Rainbow Wrack captured with underwater camera from (S1) Sidi Bouzid coast near Eljadida city at $5 m$ depth. Picture: Younes Boundir (C) June 2018

\section{Biochemical and physiological parameters}

Chlorophyll

The contents of chlorophyll a, c were estimated using the method of Jeffrey and Humphrey (1975). Pigment concentrations are expressed as $\mu \mathrm{g} / \mathrm{g}$ FW (Fresh Weight) and calculated according to the following equations: 


$$
\begin{aligned}
& \text { Chlorophyll } \mathrm{a}=11.47 \times \text { O. D. } 664-0.40 \times \text { O. D. } 630 \\
& \text { Chlorophyll } \mathrm{c}=24.36 \times \text { O. D. } 630-3.73 \times \text { O. D. } 664
\end{aligned}
$$

The pigments were extracted from $1 \mathrm{~g}$ seaweed Bushy Rainbow Wrack with $10 \mathrm{~mL}$ of acetone in the presence of calcium carbonate. Then put in $4^{\circ} \mathrm{C}$ for 24 hours in the dark. The chlorophyll content was determined in triplicate.

Total carotenoids were calculated according to the equation of Lichtenthaler (1987):

$$
\text { Carotenoid }=\frac{1000 \times \text { 0. D. } 470-1.9 \times \text { Cha }-63.14 \mathrm{Chb}}{214}
$$

\section{Glycinebetaine $(G B)$}

Analysis of GB was performed according to the method of Grieve and Grattan (1986). $0.5 \mathrm{~g}$ DW (dry weight) of the seaweed Bushy Rainbow Wrack prepared in $20 \mathrm{~mL}$ test tubes was mechanically shaken with $20 \mathrm{~mL}$ of deionized $\mathrm{H}_{2} \mathrm{O}$ at $25^{\circ} \mathrm{C}$ for $24 \mathrm{~h}$. The extracts then were diluted $1: 1$ with $2 \mathrm{~N} \mathrm{H}_{2} \mathrm{SO}_{4} .0 .5 \mathrm{ml}$ of this solution were measured into heavy walled glass centrifuge tubes and cooled in ice water for about 1 hour. A preparation of $0.2 \mathrm{ml} \mathrm{KI-I2}$ was made by dissolving $20 \mathrm{~g}$ of KI and $15.7 \mathrm{~g}$ of iodine in $100 \mathrm{ml}$. Then, water was added and the reactants were gently stirred with a vortex mixer. The tubes were stored for $16 \mathrm{~h}$ at $0-4^{\circ} \mathrm{C}$ and then centrifuged at $10000 \mathrm{rpm}$ at $0^{\circ} \mathrm{C}$ for $15 \mathrm{~min}$. The supernatant was aspirated and dissolved in $9 \mathrm{ml}$ of 1,2-dichloroethane. Vortex mixing was made to effect complete solution in the developing solvent. The absorbance was measured after $2 \mathrm{~h}$ at $365 \mathrm{~nm}$. The concentration was estimated by using a standard curve developed with different concentration of GB in triplicate.

\section{Proline}

The method used is the one of Monneveux and Nemmar (1986). $100 \mathrm{mg}$ of the seaweed Bushy Rainbow Wrack are directly weighed and then placed in a test tube. $2 \mathrm{ml}$ of methanol $40 \%$ is added to the tubes and placed in a water bath at $85^{\circ} \mathrm{C}$ for about 1 hour. $1 \mathrm{ml}$ of the solution is added to $25 \mathrm{mg}$ ninhydrin after cooling, $1 \mathrm{ml}$ of acetic acid and $1 \mathrm{ml}$ of the mixture distilled water-acetic, acid-acetic, acid-acid orthophosphoric, of density $1.7(120,300,80: \mathrm{v} / \mathrm{v} / \mathrm{v})$. The solution is brought again for 30 minutes in a water bath at $100^{\circ} \mathrm{C}$, then cooled and added to $5 \mathrm{ml}$ of toluene. After agitation, a pinch of $\mathrm{Na}_{2} \mathrm{SO}_{4}$ is added to each tube. Absorbance was measured at $528 \mathrm{~nm}$.

\section{Total phenolic compounds (TPC)}

TPC of the extract was estimated by the method of Taga et al. (1984). Dry weight samples of the seaweed Bushy Rainbow Wrack were prepared in 60:40 $(0.3 \% \mathrm{HCl})$ acidified methanol/water. Solutions of $100 \mu \mathrm{L}$ were added to $2 \mathrm{~mL}$ of $\mathrm{Na}_{2} \mathrm{CO}_{3}(2 \%)$. $100 \mu \mathrm{L}$ of Folin-Ciocalteau (50\%) reagent were added after 2 min. Absorbance was measured after 2 hours at $750 \mathrm{~nm}$. Gallic acid standards was prepared with a range of concentrations of $10 \mathrm{mg} / \mathrm{mL}$ to $200 \mathrm{mg} / \mathrm{mL}$. The phenolic concentrations were determined by comparison with the standard calibration curve. Phenolic content was expressed as gallic acid equivalent (GAE). 


\section{Statistical analysis}

All the analyzed parameters were established in triplicate and gave mean values and standard deviation. The values were tested for normality and homogeneity of variance, as well as for significance between parameters using a one-way and two-way analysis of variance with Excel 2016 and SPSS (IBM, USA), version 22. Correlation matrix was used to determine the relation between the physiological parameters, Heavy metals and the physicochemical compounds studied. Principal Component Analysis (PCA) was performed using SPSS (IBM, USA), version 22.

\section{Results}

\section{Distribution of Bushy Rainbow Wrack}

The preliminary study shows that there is a first presence of Bushy Rainbow Wrack in the control station (S1) at Eljadida coast during the 4 seasons. Essaouira city comes in the second range where this specie is more present in (S5). Safi coast comes in the thirth place where the Bushy Rainbow Wrack is abondant in unpolluted station (S3) (Table 1).

Table 1. Bushy Rainbow Wrack inventory in the studied areas during autumn-summer 2018

\begin{tabular}{|c|c|c|c|c|c|}
\hline City & Station & Autumn & Winter & Spring & Summer \\
\hline \multirow{2}{*}{ Eljadida } & S1 & A & $\mathrm{A}$ & $\mathrm{A}$ & A \\
\hline & S2 & - & - & - & - \\
\hline \multirow{3}{*}{ Safi } & S3 & $\mathrm{R}$ & $\mathrm{R}$ & $\mathrm{R}$ & $\mathrm{R}$ \\
\hline & S4 & - & - & - & - \\
\hline & S5 & - & - & - & - \\
\hline \multirow{3}{*}{ Essaouira } & S6 & $\mathrm{R}$ & $\mathrm{R}$ & $\mathrm{F}$ & $\mathrm{A}$ \\
\hline & S7 & - & - & $\mathrm{R}$ & F \\
\hline & S8 & - & - & $\mathrm{R}$ & $\mathrm{F}$ \\
\hline
\end{tabular}

-: Absence of Bushy Rainbow Wrack. A: Abundant, F: Frequent, R: Rare

\section{Physicochemical parameters: nitrogenous and phosphorus compounds}

Statistical analysis of nitrogenous compounds didn't show significant difference between control and polluted stations $(\mathrm{F}=11.29 ; \mathrm{p}<0.01)$. Thus, it could not explain the degradation of the species studied. Total nitrogenous concentrations are ranged between $2.52 \pm 0.30$ and $4.70 \pm 0.16 \mathrm{mg} / \mathrm{L}$ and those of nitrate between $1.30 \pm 0.56$ and $2.56 \pm 0.46 \mathrm{mg} / \mathrm{L}$ (Table 2).

Statistical analyses of phosphorus compounds, however, have showed a highly significant difference between the areas studied $(\mathrm{F}=1.65 ; \mathrm{p}<<0.01)$. The maximum total phosphate concentration was recorded in the Phosphate industrial area (S5), at Safi coast with $2.00 \pm 0.13 \mathrm{mg} / \mathrm{L}$ and the minimum in the control station (S1), in Sidi Bouzid coast with only $0.04 \pm 0.01 \mathrm{mg} / \mathrm{L}$ (Table 2 ).

\section{Heavy metals analysis}

\section{Heavy metals analysis in seawater}

The analysis of metals shows that the mean metal levels in seawater decreased in the following order: $\mathrm{Pb}>\mathrm{Cd}>\mathrm{Cr}>\mathrm{Cu}$ (Table 3). The results obtained are discussed on the basis of the Moroccan standards for direct discharges of heavy metals into aquatic 
environments (surface water). The maximum concentrations required by the above standard are: $0.5 \mathrm{mg} / \mathrm{l}$ for $\mathrm{Pb}, 2 \mathrm{mg} / \mathrm{l}$ for $\mathrm{Cr}, 0.5 \mathrm{mg} / \mathrm{l}$ for $\mathrm{Cu}$ and $0.2 \mathrm{mg} / \mathrm{l}$ for $\mathrm{Cd}$ (FAO, 2006). The highest concentrations of toxic metals were recorded in two polluted stations near to Safi coast: a fish industrial discharges (Cd: $1.12 \pm 0.15 \mu \mathrm{g} / \mathrm{L} ; \mathrm{Pb}: 2.563 \pm 0.73 \mu \mathrm{g} / \mathrm{L})$ and phosphate discharge area $(\mathrm{Cd}: 1.15 \pm 0.88 \mu \mathrm{g} / \mathrm{L} ; \mathrm{Pb}: 1.53 \pm 0.55 \mu \mathrm{g} / \mathrm{L})$.

Table 2. Variation of Phosphorus and Nitrogenous compounds in different stations

\begin{tabular}{|c|c|c|c|c|c|c|c|c|}
\hline Station & eason & TP & DP & $\mathrm{PO}_{4}{ }^{3-}$ & TN & DN & $\mathrm{NO}_{3}{ }^{-}$ & $\mathrm{NH}_{4}{ }^{+}$ \\
\hline \multirow{4}{*}{ S1 } & tumn & $0.06 \pm 0.03^{\mathrm{C}^{*}}$ & $0.05 \pm 0.02^{\mathrm{C}}$ & $0.02 \pm 0.01^{\mathrm{C}}$ & $4.03 \pm 0.14^{\mathrm{A}}$ & $3.74 \pm 0.11^{\mathrm{A}}$ & $2.14 \pm 0.20^{\mathrm{A}}$ & $0.89 \pm 0.09$ \\
\hline & inter & $0.07 \pm 0.02^{\mathrm{C}}$ & $0.08 \pm 0.03^{\mathrm{BC}}$ & $0.05 \pm 0.02^{\mathrm{C}}$ & $4.40 \pm 0.13^{\mathrm{A}}$ & $4.10 \pm 0.14^{\mathrm{A}}$ & $2.40 \pm 0.16^{\mathrm{A}}$ & $1.14 \pm 0.04^{\mathrm{A}}$ \\
\hline & & & $0.07 \pm 0.02^{\mathrm{B}}$ & $0.04 \pm 0.01^{\mathrm{C}}$ & $4.31 \pm 0.14^{\mathrm{A}}$ & $3.65 \pm 0.13^{\mathrm{A}}$ & $2.33 \pm 0.14^{\mathrm{A}}$ & $0.90 \pm 0.05^{\mathrm{BC}}$ \\
\hline & & & $0.05 \pm 0.03^{\mathrm{C}}$ & $0.03 \pm 0.01^{\mathrm{C}}$ & $4.12 \pm 0.15^{\mathrm{A}}$ & $3.21 \pm 0.12^{\mathrm{A}}$ & $2.20 \pm 0.12^{\mathrm{A}}$ & $1.11 \pm$ \\
\hline \multirow{4}{*}{$\mathbf{S}$} & & & $0.05 \pm 0.02^{\mathrm{C}}$ & $0.05 \pm 0.02^{\mathrm{C}}$ & $2.15 \pm 0.12^{\mathrm{C}}$ & $1.23 \pm 0.21^{\mathrm{B}}$ & $1.30 \pm 0.56^{\mathrm{B}}$ & $0.74 \pm 0.16^{\mathrm{AB}}$ \\
\hline & MICT & $0.09 \pm 0.05^{\mathrm{C}}$ & $0.08 \pm 0.01^{\mathrm{BC}}$ & $0.07 \pm 0.03^{\mathrm{BC}}$ & $2.40 \pm 0.14^{\mathrm{C}}$ & $2.30 \pm 0.23^{\mathrm{B}}$ & $1.50 \pm 0.20^{\mathrm{B}}$ & $1.12 \pm 0.13^{\mathrm{A}}$ \\
\hline & & & $0.07 \pm 0.03^{\mathrm{B}}$ & $0.06 \pm 0.02^{\mathrm{BC}}$ & $2.32 \pm 0.15^{\mathrm{C}}$ & $1.96 \pm 0.17^{\mathrm{B}}$ & $1.45 \pm 0.23^{\mathrm{B}}$ & $1.10 \pm 0.16^{\mathrm{A}}$ \\
\hline & & $0.05=$ & $0.04 \pm 0.01^{\mathrm{C}}$ & $0.06 \pm 0.01^{\mathrm{BC}}$ & $2.30 \pm 0.13^{\mathrm{C}}$ & $1.50 \pm 0.12^{\mathrm{B}}$ & $1.33 \pm 0.12^{\mathrm{B}}$ & $0.88 \pm 0.18^{\mathrm{A}}$ \\
\hline & & $0.26 \pm$ & $0.06 \pm 0.03^{\mathrm{C}}$ & $0.03 \pm 0.01^{\mathrm{C}}$ & 2.65 & $1.65 \pm 0.18^{\mathrm{B}}$ & $1.70 \pm 0.13^{\mathrm{AB}}$ & $1.15 \pm 0.13^{\mathrm{A}}$ \\
\hline & & 0.38 & $0.08 \pm 0.03^{\mathrm{BC}}$ & $0.05 \pm 0$ & $2.90 \pm$ & $2.40 \pm 0.17^{\mathrm{B}}$ & $1.90 \pm 0.1$ & $1.21 \pm 0.18^{\mathrm{A}}$ \\
\hline & Spring & $0.25=$ & $0.05 \pm 0.02^{\mathrm{C}}$ & $0.04 \pm 0.01^{\mathrm{C}}$ & $2.85 \pm 0.15^{\mathrm{BC}}$ & $1.96 \pm 0.13^{\mathrm{B}}$ & $1.88 \pm 0.18^{\mathrm{AB}}$ & $1.12 \pm 0.22^{\mathrm{A}}$ \\
\hline & & 0.2 & $0.06 \pm$ & $0.03 \pm 0.01^{\mathrm{C}}$ & $2.74 \pm$ & $1.80 \pm 0.12^{\mathrm{B}}$ & $1.65 \pm 0.14^{\mathrm{B}}$ & $1.10 \pm 0.17^{\mathrm{A}}$ \\
\hline \multirow{4}{*}{ S4 } & & & $0.06 \pm$ & $0.05 \pm 0.02^{\mathrm{C}}$ & $4.33 \pm 0.18^{\mathrm{A}}$ & $1.78 \pm 0.13^{\mathrm{B}}$ & $2.22 \pm 0.25^{\mathrm{A}}$ & $0.54 \pm 0.18^{\mathrm{B}}$ \\
\hline & & $0.29 \pm$ & $0.10 \pm 0.03^{\mathrm{AB}}$ & $0.07 \pm 0.02^{\mathrm{BC}}$ & $4.70 \pm 0.16^{\mathrm{A}}$ & $2.60 \pm 0.14^{\mathrm{B}}$ & $2.40 \pm 0.24^{\mathrm{A}}$ & $0.99 \pm 0.17^{\mathrm{AB}}$ \\
\hline & & 0.13 & $0.10 \pm 0$ & $0.06 \pm 0.01^{\mathrm{BC}}$ & $4.62 \pm 0.12^{\mathrm{A}}$ & $2.20 \pm 0.14^{\mathrm{B}}$ & $2.33 \pm 0.22^{\mathrm{A}}$ & $0.85 \pm 0.18^{\mathrm{AB}}$ \\
\hline & & $0.15=$ & $0.05 \pm$ & $0.06 \pm 0.02^{\mathrm{BC}}$ & $4.56 \pm 0.13^{\mathrm{A}}$ & $1.96 \pm 0.15^{\mathrm{B}}$ & $2.10 \pm 0.14^{\mathrm{A}}$ & $1.23 \pm 0.13^{\mathrm{A}}$ \\
\hline \multirow{4}{*}{ S5 } & & & & & & & & \\
\hline & & 2.00 & $1.58 \pm 0.06^{\mathrm{A}}$ & $0.11 \pm 0.02^{\mathrm{A}}$ & $3.10 \pm 0.14^{\mathrm{B}}$ & $3.00 \pm 0.17^{\mathrm{A}}$ & $2.60 \pm 0.45^{\mathrm{A}}$ & $0.65 \pm 0.17^{\mathrm{B}}$ \\
\hline & & $1.22 \pm$ & $1.15 \pm 0.08^{\mathrm{A}}$ & $0.11 \pm 0.03^{\mathrm{A}}$ & $2.63 \pm 0.17^{\mathrm{B}}$ & $2.80 \pm 0.20^{\mathrm{A}}$ & $2.56 \pm 0.46^{\mathrm{A}}$ & $0.24 \pm 0.18^{\mathrm{C}}$ \\
\hline & & & 1.22 & $0.09 \pm$ & & $2.55 \pm 0.13^{\mathrm{A}}$ & $2.41 \pm 0.34^{\mathrm{A}}$ & $0.32 \pm 0.13^{\mathrm{C}}$ \\
\hline \multirow{4}{*}{ S6 } & & 0.2 & $0.03 \pm 0.01^{\mathrm{C}}$ & $0.03 \pm 0$ & $3.21 \pm$ & $1.80 \pm 0.12^{\mathrm{AB}}$ & $1.46=$ & 0.54 \\
\hline & & 0.48 & 0.05 & $0.05 \pm$ & $4.20=$ & $2.00 \pm 0.16^{\mathrm{B}}$ & $1.90 \pm 0.12^{\mathrm{B}}$ & $1.21 \pm 0.12^{\mathrm{A}}$ \\
\hline & & 0.3 & $0.01 \pm$ & $0.04 \pm 0.01^{\mathrm{C}}$ & $4.10 \pm 0.13^{\mathrm{A}}$ & $1.55 \pm 0.13^{\mathrm{B}}$ & $1.56 \pm 0.11^{\mathrm{B}}$ & $0.74 \pm 0.16^{\mathrm{AB}}$ \\
\hline & & 0.35 & $0.02 \pm 0.01^{\mathrm{C}}$ & $0.04 \pm 0.02^{\mathrm{C}}$ & $3.32 \pm 0.12^{\mathrm{B}}$ & $1.69 \pm 0.17^{\mathrm{B}}$ & $1.70 \pm 0.10^{\mathrm{B}}$ & $0.64 \pm 0.17^{\mathrm{AB}}$ \\
\hline \multirow{4}{*}{ S7 } & & 0.24 & & & 2.1 & 2.54 & 2.22 & $0.45 \pm 0.15^{\mathrm{B}}$ \\
\hline & & $0.30 \pm 0.01^{\mathrm{B}}$ & $0.17 \pm 0.03^{\mathrm{B}}$ & $0.07 \pm 0.02^{\mathrm{B}}$ & $2.90 \pm 0.15^{\mathrm{AB}}$ & $2.60 \pm 0.13^{\mathrm{A}}$ & $2.50 \pm 0.14^{\mathrm{A}}$ & $0.87 \pm 0.12^{\mathrm{AB}}$ \\
\hline & & $0.25 \pm 0.01^{\mathrm{B}}$ & $0.12 \pm 0.04^{\mathrm{B}}$ & $0.07 \pm 0.01^{\mathrm{B}}$ & $2.55 \pm 0.13^{\mathrm{B}}$ & $2.33 \pm 0.14^{\mathrm{A}}$ & $2.45 \pm 0.11^{\mathrm{A}}$ & $0.32 \pm 0.14^{\mathrm{C}}$ \\
\hline & & & $0.14 \pm 0.06^{\mathrm{B}}$ & $0.06 \pm 0.02^{\mathrm{BC}}$ & $2.31 \pm 0.12^{\mathrm{C}}$ & $2.30 \pm 0.18^{\mathrm{A}}$ & $2.30 \pm 0.24^{\mathrm{A}}$ & $0.35 \pm 0.12^{\mathrm{C}}$ \\
\hline \multirow{4}{*}{ S8 } & & $0.26 \pm 0.03^{\mathrm{B}}$ & $0.21 \pm 0.02^{\mathrm{B}}$ & $0.11 \pm 0.01^{\mathrm{A}}$ & $3.31 \pm 0.16^{\mathrm{B}}$ & $2.50 \pm 0.12^{\mathrm{A}}$ & $1.30 \pm 0.12^{\mathrm{BC}}$ & $0.58 \pm 0.13^{\mathrm{BC}}$ \\
\hline & ter & $0.36 \pm 0.06^{\mathrm{B}}$ & $0.23 \pm 0.03^{\mathrm{B}}$ & $0.11 \pm 0.02^{\mathrm{A}}$ & $4.30 \pm 0.14^{\mathrm{A}}$ & $2.80 \pm 0.21^{\mathrm{A}}$ & $1.50 \pm 0.13^{\mathrm{B}}$ & $0.65 \pm 0.13^{\mathrm{BC}}$ \\
\hline & & $0.26 \pm 0.03^{\mathrm{B}}$ & $0.16 \pm 0.02^{\mathrm{B}}$ & $0.11 \pm 0.01^{\mathrm{A}}$ & $4.23 \pm 0.16^{\mathrm{A}}$ & $2.66 \pm 0.23^{\mathrm{A}}$ & $1.45 \pm 0.23^{\mathrm{AB}}$ & $0.67 \pm 0.16^{\mathrm{B}}$ \\
\hline & Summer & $0.25 \pm 0.05^{\mathrm{B}}$ & $0.19 \pm 0.01^{\mathrm{B}}$ & $0.11 \pm 0.02^{\mathrm{A}}$ & $3.56 \pm 0.12^{\mathrm{A}}$ & $2.12 \pm 0.18^{\mathrm{A}}$ & $1.36 \pm 0.14^{\mathrm{AB}}$ & $0.54 \pm 0.11^{\mathrm{B}}$ \\
\hline
\end{tabular}

$*$ The different upper-case letters in the same row indicate the differences between the studied stations and seasons at the level $(\mathrm{p}<0.05)$. TP: Total Phosphorus; DP: Dissolved Phosphorus; $\mathrm{PO}_{4}{ }^{3-}$ : Orthophosphate; TN: Total Nitrogenous; DN: Dissolved Nitrogenous; $\mathrm{NO}_{3}{ }^{-}$: Nitrate; $\mathrm{NH}_{4}{ }^{+}$: $\mathrm{Ammonium}$

\section{Heavy metals analysis in Bushy Rainbow Wrack}

The analysis of heavy metals shows that the mean levels in seaweed Bushy Rainbow Wrack decreased in the following order: $\mathrm{Cu}>\mathrm{Pb}>\mathrm{Cr}>\mathrm{Cd}$. The results obtained are discussed on the basis of comparaison of these heavy metals levels in seaweed Cystoseira sp. from other different locations in the world. The highest concentrations of all heavy metal were recorded in the polluted area (S8), the port station at Essaouira coast (Cd: $2.60 \pm 0.15 \mu \mathrm{g} / \mathrm{g}$ during spring; $\mathrm{Pb}$ : $4.43 \pm 0.73 \mu \mathrm{g} / \mathrm{g}$ during autumn, $\mathrm{Cu}: 10.60 \pm 1.14 \mu \mathrm{g} / \mathrm{g}$ during summer and Cr: $2.90 \pm 0.23 \mu \mathrm{g} / \mathrm{g}$ during autumn) (Table 4). 
Table 3. Heavy metals mean concentrations of $\mathrm{Cd}, \mathrm{Pb}, \mathrm{Cu}$, and $\mathrm{Cr}$ in seawater along the studied stations

\begin{tabular}{c|c|c|c|c}
\hline Station & Cd & Pb & Cu & Cr \\
\hline S1 & $0.09 \pm 0.02^{\mathrm{C} *}$ & $0.93 \pm 0.28^{\mathrm{B}}$ & $0.08 \pm 0.02^{\mathrm{C}}$ & $0.07 \pm 0.02^{\mathrm{C}}$ \\
S2 & $0.11 \pm 0.04^{\mathrm{BC}}$ & $0.98 \pm 0.27^{\mathrm{B}}$ & $0.12 \pm 0.07^{\mathrm{C}}$ & $0.09 \pm 0.03^{\mathrm{C}}$ \\
S3 & $0.17 \pm 0.03^{\mathrm{B}}$ & - & $0.19 \pm 0.06^{\mathrm{C}}$ & $0.25 \pm 0.06^{\mathrm{BC}}$ \\
S4 & $1.12 \pm 0.15^{\mathrm{A}}$ & $2.56 \pm 0.73^{\mathrm{A}}$ & $0.20 \pm 0.04^{\mathrm{BC}}$ & $0.98 \pm 0.17^{\mathrm{A}}$ \\
S5 & $1.15 \pm 0.88^{\mathrm{A}}$ & $1.53 \pm 0.55^{\mathrm{A}}$ & $0.60 \pm 0.17^{\mathrm{A}}$ & $0.84 \pm 0.07^{\mathrm{A}}$ \\
S6 & $0.12 \pm 0.07^{\mathrm{B}}$ & $1.12 \pm 0.24^{\mathrm{AB}}$ & $0.09 \pm 0.04^{\mathrm{C}}$ & $0.03 \pm 0.01^{\mathrm{C}}$ \\
S7 & $0.15 \pm 0.09^{\mathrm{B}}$ & $0.97 \pm 0.26^{\mathrm{B}}$ & $0.16 \pm 0.08^{\mathrm{C}}$ & $0.04 \pm 0.02^{\mathrm{C}}$ \\
S8 & $0.17 \pm 0.06^{\mathrm{B}}$ & $0.87 \pm 0.19^{\mathrm{B}}$ & $0.13 \pm 0.03^{\mathrm{C}}$ & $0.05 \pm 0.01^{\mathrm{C}}$ \\
\hline
\end{tabular}

*The different upper-case letters in the same row indicate the differences between the studied stations and seasons at the level $(\mathrm{p}<0.05)$

Table 4. Heavy metal mean concentrations ( $\mu \mathrm{g} / \mathrm{g}$ Dry Weight) of $\mathrm{Cd}, \mathrm{Pb}, \mathrm{Cu}$, and Cr in Bushy Rainbow Wrack collected during autumn - summer 2018 along the stations studied

\begin{tabular}{|c|c|c|c|c|c|}
\hline Station & Season & $\overline{C d}$ & $\mathbf{P b}$ & $\mathbf{C u}$ & $\mathrm{Cr}$ \\
\hline \multirow{4}{*}{ S1 } & Autumn & $0.10 \pm 0.02^{\mathrm{C}^{*}}$ & $2.30 \pm 0.32^{\mathrm{AB}}$ & $0.21 \pm 0.05^{\mathrm{C}}$ & $0.11 \pm 0.02^{\mathrm{C}}$ \\
\hline & Winter & $0.22 \pm 0.05^{\mathrm{BC}}$ & $1.20 \pm 0.08^{\mathrm{C}}$ & $3.07 \pm 0.19^{\mathrm{BC}}$ & $0.20 \pm 0.05^{\mathrm{C}}$ \\
\hline & Spring & $0.39 \pm 0.09^{\mathrm{BC}}$ & $1.30 \pm 0.05^{\mathrm{C}}$ & $0.36 \pm 0.05^{\mathrm{C}}$ & $0.36 \pm 0.03^{\mathrm{C}}$ \\
\hline & Summer & $0.10 \pm 0.03^{\mathrm{C}}$ & $0.50 \pm 0.04^{\mathrm{C}}$ & $0.17 \pm 0.09^{\mathrm{C}}$ & $0.14 \pm 0.01^{\mathrm{C}}$ \\
\hline \multirow{4}{*}{$\mathbf{S 2}$} & Autumn & - & - & - & - \\
\hline & Winter & - & - & - & - \\
\hline & Spring & - & - & - & - \\
\hline & Summer & - & - & - & - \\
\hline \multirow{4}{*}{$\mathbf{S 3}$} & Autumn & $0.20 \pm 0.06^{\mathrm{C}}$ & $2.51 \pm 0.08^{\mathrm{B}}$ & $4.00 \pm 0.18^{\mathrm{BC}}$ & $0.14 \pm 0.04^{\mathrm{C}}$ \\
\hline & Winter & $0.42 \pm 0.05^{\mathrm{BC}}$ & $2.14 \pm 0.04^{\mathrm{BC}}$ & $2.72 \pm 0.07^{\mathrm{C}}$ & $0.25 \pm 0.06^{\mathrm{C}}$ \\
\hline & Spring & $0.17 \pm 0.04^{\mathrm{BC}}$ & $1.25 \pm 0.08^{\mathrm{C}}$ & $0.45 \pm 0.05^{\mathrm{C}}$ & $0.24 \pm 0.03^{\mathrm{C}}$ \\
\hline & Summer & $0.20 \pm 0.02^{\mathrm{C}}$ & $1.22 \pm 0.06^{\mathrm{C}}$ & $1.90 \pm 0.09^{\mathrm{C}}$ & $0.36 \pm 0.02^{\mathrm{C}}$ \\
\hline \multirow{4}{*}{ S4 } & Autumn & - & - & - & - \\
\hline & Winter & - & - & - & - \\
\hline & Spring & - & - & - & - \\
\hline & Summer & - & - & - & - \\
\hline \multirow{4}{*}{ S5 } & Autumn & - & - & - & - \\
\hline & Winter & - & - & - & - \\
\hline & Spring & - & - & - & - \\
\hline & Summer & - & - & - & - \\
\hline \multirow{4}{*}{ S6 } & Autumn & $0.50 \pm 0.07^{\mathrm{C}}$ & $1.20 \pm 0.06^{\mathrm{C}}$ & $2.10 \pm 0.14^{\mathrm{C}}$ & $0.28 \pm 0.04^{\mathrm{C}}$ \\
\hline & Winter & $0.60 \pm 0.05^{\mathrm{C}}$ & $2.00 \pm 0.05^{\mathrm{BC}}$ & $4.55 \pm 0.08^{\mathrm{B}}$ & $0.58 \pm 0.08^{\mathrm{BC}}$ \\
\hline & Spring & $0.20 \pm 0.06^{\mathrm{C}}$ & $1.13 \pm 0.09^{\mathrm{C}}$ & $4.10 \pm 0.05^{\mathrm{B}}$ & $0.26 \pm 0.05^{\mathrm{C}}$ \\
\hline & Summer & $0.15 \pm 0.04^{\mathrm{C}}$ & $1.11 \pm 0.06^{\mathrm{C}}$ & $6.10 \pm 0.10^{\mathrm{B}}$ & $0.12 \pm 0.07^{\mathrm{C}}$ \\
\hline \multirow{4}{*}{ S7 } & Autumn & $1.22 \pm 0.13^{\mathrm{B}}$ & $3.20 \pm 0.45^{\mathrm{A}}$ & $4.62 \pm 0.12^{\mathrm{B}}$ & $2.66 \pm 0.13^{\mathrm{A}}$ \\
\hline & Winter & $1.21 \pm 0.18^{\mathrm{B}}$ & $1.05 \pm 0.07^{\mathrm{C}}$ & $5.78 \pm 0.13^{\mathrm{B}}$ & $0.98 \pm 0.11^{\mathrm{BC}}$ \\
\hline & Spring & $1.90 \pm 0.17^{\mathrm{AB}}$ & $2.25 \pm 0.23^{\mathrm{B}}$ & $7.50 \pm 0.07^{\mathrm{B}}$ & $1.85 \pm 0.13^{\mathrm{B}}$ \\
\hline & Summer & $1.70 \pm 0.16^{\mathrm{AB}}$ & $2.20 \pm 0.30^{\mathrm{B}}$ & $8.80 \pm 0.09^{\mathrm{A}}$ & $1.50 \pm 0.18^{\mathrm{B}}$ \\
\hline \multirow{4}{*}{ S8 } & Autumn & $2.50 \pm 0.15^{\mathrm{A}}$ & $4.43 \pm 0.73^{\mathrm{A}}$ & $5.60 \pm 0.11^{\mathrm{BC}}$ & $2.90 \pm 0.23^{\mathrm{A}}$ \\
\hline & Winter & $2.26 \pm 0.13^{\mathrm{A}}$ & $1.90 \pm 0.20^{\mathrm{AB}}$ & $4.32 \pm 1.23^{\mathrm{BC}}$ & $1.99 \pm 0.16^{\mathrm{A}}$ \\
\hline & Spring & $2.60 \pm 0.15^{\mathrm{A}}$ & $2.54 \pm 0.32^{\mathrm{AB}}$ & $10.41 \pm 1.56^{\mathrm{A}}$ & $2.23 \pm 0.17^{\mathrm{A}}$ \\
\hline & Summer & $1.60 \pm 0.13^{\mathrm{B}}$ & $1.90 \pm 0.24^{\mathrm{AB}}$ & $10.60 \pm 1.14^{\mathrm{A}}$ & $2.89 \pm 0.18^{\mathrm{A}}$ \\
\hline
\end{tabular}

-: Absence of Bushy Rainbow Wrack.

*The different upper-case letters in the same row indicate the differences between the studied stations and seasons at the level $(\mathrm{p}<0.05)$ 


\section{Biochemical and physiological parameters}

\section{Chlorophyll contents}

Maximum value of chlorophyll a were recorded at (S1) Sidi Bouzid coast near Eljadida city with $586 \pm 167 \mu \mathrm{g} / \mathrm{g}$ FW during spring season, and reached its minimum at (S8) The port station in Essaouira city with $59 \pm 14 \mu \mathrm{g} / \mathrm{g}$ FW during autumn season. Values of chlorophyll c has its maximum as well at (S1) Sidi Bouzid coast near Eljadida city with $387 \pm 93 \mu \mathrm{g} / \mathrm{g}$ FW during spring and summer season, and its minimum also at (S8) The port station in Essaouira city with $34 \pm 13 \mu \mathrm{g} / \mathrm{g}$ FW during spring season. The same thing for carotenoid that shown a maximum value at (S1) Sidi Bouzid coast near Eljadida city with $399 \pm 175 \mu \mathrm{g} / \mathrm{g}$ FW during summer season and a minimum likewise at (S8) The port station in Essaouira city with $34 \pm 0.9 \mu \mathrm{g} / \mathrm{g}$ FW during summer season (Table 5).

Table 5. Concentrations of Chlorophyll a, c, carotenoid and proline ( $\mu \mathrm{g} / \mathrm{g}$ Fresh Weight), GB and TPC ( $\mathrm{mg} / \mathrm{g}$ Dry Weight) of Bushy Rainbow Wrack in different stations during the 4 seasons of 2018

\begin{tabular}{|c|c|c|c|c|c|c|c|}
\hline Station & Season & Chl a & Chl c & Carotenoid & Proline & GB & TPC \\
\hline \multirow{4}{*}{ S1 } & Autumn & $475 \pm 133^{\mathrm{A}^{*}}$ & $267 \pm 97^{\mathrm{AB}}$ & $255 \pm 76^{\mathrm{A}}$ & $69.06 \pm 24.22^{\mathrm{C}}$ & $2.06 \pm 0.25^{\mathrm{C}}$ & $0.56 \pm 0.14^{\mathrm{C}}$ \\
\hline & Winter & $567 \pm 128^{\mathrm{A}}$ & $267 \pm 86^{\mathrm{AB}}$ & $276 \pm 94^{\mathrm{A}}$ & $69.06 \pm 13.03^{\mathrm{C}}$ & $2.06 \pm 0.53^{\mathrm{C}}$ & $0.52 \pm 0.12^{\mathrm{C}}$ \\
\hline & Spring & $586 \pm 167^{\mathrm{A}}$ & $387 \pm 93^{\mathrm{A}}$ & $376 \pm 146^{\mathrm{A}}$ & $71.94 \pm 18.13^{C}$ & $2.15 \pm 0.33^{\mathrm{C}}$ & $0.56 \pm 0.23^{\mathrm{C}}$ \\
\hline & Summer & $489 \pm 120^{\mathrm{A}}$ & $387 \pm 56^{\mathrm{A}}$ & $399 \pm 175^{\mathrm{A}}$ & $112.23 \pm 14.50^{\mathrm{BC}}$ & $3.35 \pm 0.45^{\mathrm{C}}$ & $0.73 \pm 0.15^{\mathrm{C}}$ \\
\hline \multirow{4}{*}{ S2 } & Autumn & - & - & - & - & - & - \\
\hline & Winter & - & - & - & - & - & - \\
\hline & Spring & - & - & - & - & - & - \\
\hline & Summer & - & - & - & - & - & - \\
\hline \multirow{4}{*}{ S3 } & Autumn & $393 \pm 117^{\mathrm{AB}}$ & $143 \pm 75^{\mathrm{AB}}$ & $129 \pm 87^{\mathrm{B}}$ & $57.55 \pm 15.11^{\mathrm{C}}$ & $1.72 \pm 0.12^{\mathrm{C}}$ & $0.72 \pm 0.17^{\mathrm{C}}$ \\
\hline & Winter & $398 \pm 128^{\mathrm{AB}}$ & $176 \pm 86^{\mathrm{AB}}$ & $175 \pm 95^{\mathrm{B}}$ & $120.86 \pm 45.16^{\mathrm{B}}$ & $3.61 \pm 0.15^{\mathrm{C}}$ & $0.74 \pm 0.14^{\mathrm{C}}$ \\
\hline & Spring & $498 \pm 127^{\mathrm{A}}$ & $198 \pm 95^{\mathrm{AB}}$ & $176 \pm 84^{\mathrm{B}}$ & $94.96 \pm 34.00^{\mathrm{BC}}$ & $2.83 \pm 0.45^{\mathrm{C}}$ & $0.68 \pm 0.12^{\mathrm{C}}$ \\
\hline & Summer & $373 \pm 57^{\mathrm{AB}}$ & $187 \pm 97^{\mathrm{AB}}$ & $183 \pm 77^{\mathrm{AB}}$ & $112.23 \pm 43.12^{\mathrm{B}}$ & $3.35 \pm 0.36^{\mathrm{C}}$ & $0.72 \pm 0.16^{\mathrm{C}}$ \\
\hline \multirow{4}{*}{ S4 } & Autumn & - & - & - & - & - & - \\
\hline & Winter & - & - & - & - & - & - \\
\hline & Spring & - & - & - & - & - & - \\
\hline & Summer & - & - & - & - & - & - \\
\hline \multirow{4}{*}{ S5 } & Autumn & - & - & - & - & - & - \\
\hline & Winter & - & - & - & - & - & - \\
\hline & Spring & - & - & - & - & - & - \\
\hline & Summer & - & - & - & - & - & - \\
\hline \multirow{4}{*}{ S6 } & Autumn & $487 \pm 156^{\mathrm{A}}$ & $276 \pm 74^{\mathrm{AB}}$ & $166 \pm 83^{B}$ & $92.08 \pm 15.00^{\mathrm{BC}}$ & $2.752 \pm 0.14^{\mathrm{C}}$ & $0.65 \pm 0.17^{\mathrm{C}}$ \\
\hline & Winter & $476 \pm 186^{\mathrm{A}}$ & $387 \pm 59^{\mathrm{A}}$ & $276 \pm 75^{\mathrm{A}}$ & $129.49 \pm 34.65^{\mathrm{B}}$ & $3.87 \pm 0.56^{\mathrm{C}}$ & $0.90 \pm 0.27^{\mathrm{BC}}$ \\
\hline & Spring & $492 \pm 94^{\mathrm{A}}$ & $254 \pm 122^{\mathrm{AB}}$ & $285 \pm 86^{\mathrm{A}}$ & $115.10 \pm 54.66^{\mathrm{B}}$ & $3.44 \pm 0.76^{\mathrm{C}}$ & $0.72 \pm 0.26^{\mathrm{C}}$ \\
\hline & Summer & $530 \pm 82^{\mathrm{A}}$ & $376 \pm 110^{\mathrm{A}}$ & $236 \pm 93^{A}$ & $109.35 \pm 45.56^{\mathrm{B}}$ & $3.268 \pm 1.78^{\mathrm{BC}}$ & $0.58 \pm 0.17^{\mathrm{C}}$ \\
\hline \multirow{4}{*}{ S7 } & Autumn & $183 \pm 56^{\mathrm{BC}}$ & $45 \pm 12^{\mathrm{C}}$ & $98 \pm 24^{\mathrm{B}}$ & $460.43 \pm 176.76^{\mathrm{A}}$ & $13.76 \pm 1.54^{\mathrm{AB}}$ & $1.36 \pm 0.28^{\mathrm{B}}$ \\
\hline & Winter & $287 \pm 86^{\mathrm{B}}$ & $98 \pm 16^{\mathrm{C}}$ & $176 \pm 37^{\mathrm{B}}$ & $215.82 \pm 76.44^{\mathrm{AB}}$ & $6.45 \pm 1.76^{\mathrm{B}}$ & $1.04 \pm 0.93^{\mathrm{AB}}$ \\
\hline & Spring & $287 \pm 89^{\mathrm{B}}$ & $65 \pm 18^{\mathrm{C}}$ & $111 \pm 30^{\mathrm{BC}}$ & $330.93 \pm 165.66^{\mathrm{AB}}$ & $9.89 \pm 1.77^{\mathrm{B}}$ & $1.11 \pm 0.28^{\mathrm{AB}}$ \\
\hline & Summer & $245 \pm 112^{\mathrm{B}}$ & $57 \pm 14^{\mathrm{C}}$ & $123 \pm 34^{\mathrm{BC}}$ & $503.59 \pm 164.43^{\mathrm{A}}$ & $15.05 \pm 2.65^{\mathrm{A}}$ & $1.20 \pm 0.17^{\mathrm{A}}$ \\
\hline \multirow{4}{*}{ S8 } & Autumn & $59 \pm 14^{\mathrm{C}}$ & $35 \pm 09^{\mathrm{C}}$ & $96 \pm 12^{\mathrm{C}}$ & $517.98 \pm 174.11^{\mathrm{A}}$ & $15.48 \pm 3.17^{\mathrm{A}}$ & $1.54 \pm 0.28^{\mathrm{A}}$ \\
\hline & Winter & $165 \pm 87^{\mathrm{BC}}$ & $76 \pm 29^{C}$ & $54 \pm 15^{\mathrm{C}}$ & $546.76 \pm 145.19^{\mathrm{A}}$ & $16.34 \pm 4.75^{\mathrm{A}}$ & $1.10 \pm 0.16^{\mathrm{AB}}$ \\
\hline & Spring & $156 \pm 75^{\mathrm{BC}}$ & $34 \pm 13^{\mathrm{C}}$ & $87 \pm 17^{\mathrm{C}}$ & $566.90 \pm 175^{\mathrm{A}}$ & $16.94 \pm 07.56^{\mathrm{A}}$ & $1.14 \pm 0.27^{\mathrm{A}}$ \\
\hline & Summer & $74 \pm 19^{C}$ & $76 \pm 33^{C}$ & $34 \pm 09^{\mathrm{C}}$ & $647.48 \pm 198^{\mathrm{A}}$ & $19.35 \pm 14.97^{\mathrm{A}}$ & $1.51 \pm 0.37^{\mathrm{A}}$ \\
\hline
\end{tabular}

-: Absence of Bushy Rainbow Wrack.

*The different upper-case letters in the same row indicate the differences between the studied stations and seasons at the level $(\mathrm{p}<0.05)$ 


\section{Glycinebetaine $(G B)$, proline and total phenolic compounds (TPC)}

GB parameter reaches a high value of $19.35 \pm 14.97 \mathrm{mg} / \mathrm{g}$ DW at (S8) The port station in Essaouira city during summer season, and achieved its minimum at (S3) Beddouza station near Safi city with $1.72 \pm 0.12 \mathrm{mg} / \mathrm{g}$ DW during autumn season. In the same way, the proline parameter has the same result as GB. The maximum value is recorded at (S8) The port station in Essaouira city with $647.48 \pm 198 \mu \mathrm{g} / \mathrm{g}$ DW during summer season, and a minimum value of $57.55 \pm 15.11 \mu \mathrm{g} / \mathrm{g}$ DW at (S3) Beddouza station near Safi city during autumn season. Moreover, TPC parameter has given the same result as GB and proline parameters which we have found a maximum value of $1.54 \pm 0.28 \mathrm{mg} / \mathrm{g}$ DW during autumn season at (S8) The port station in Essaouira city, as well as a minimum value of $0.52 \pm 0.12 \mathrm{mg} / \mathrm{g}$ DW at (S1) Sidi Bouzid coast near Eljadida city during winter season (Table 5).

\section{Statistical analysis}

A tow-way mixed ANOVA was conducted to investigate the impact of seasons, the sampling sites and Heavy metals concentrations in sea water. There was a significant main effect of sampling sites and seasons, $\mathrm{F}(1,66)=183.397, \mathrm{p}<0.001$ (Table 6). Moreover, there was a significant interaction between Heavy metals, Sampling sites and seasons, $\mathrm{F}(1,66)=2.197, \mathrm{p}<0.001$ (Table 7).

Table 6. Tests of Within-Subjects Contrasts

\begin{tabular}{c|c|c|c|c|c|c}
\hline Source & Seasons_Sites & $\begin{array}{c}\text { Type III Sum of } \\
\text { Squares }\end{array}$ & df & Mean Square & F & Sig. \\
\hline Seasons_Sites & Linear & 298.239 & 1 & 298.239 & 183.397 & 0.000 \\
Seasons_Sites* HM & Linear & 316.802 & 66 & 4.800 & 2.952 & 0.000 \\
Error(Seasons_Sites) & Linear & 99.198 & 61 & 1.626 & & \\
\hline
\end{tabular}

Table 7. Tests of Between-Subjects Effects

\begin{tabular}{c|c|c|c|c|c}
\hline Source & Type III Sum of Squares & df & Mean Square & F & Sig. \\
\hline Intercept & 2251.892 & 1 & 2251.892 & 1114.998 & 0.000 \\
HM & 292.802 & 66 & 4.436 & 2.197 & 0.000 \\
Error & 123.198 & 61 & 2.020 & & \\
\hline
\end{tabular}

By performing the Principal Component Analysis (PCA) on the physiological parameters (Chlorophyll a, Chlorophyll c, Carotenoid, Proline, Glycinebetaine (GB) and Total Phenolic Compounds (TPC)) combined with the physicochemical parameters (Phosphorus and Nitrogenous compounds) and heavy metals $(\mathrm{Cu}, \mathrm{Cd}, \mathrm{Pb}$ and $\mathrm{Cr}$ ) under study, two principal components have been extracted by covering $74.085 \%$ of the cumulative variance (Table 8).

\section{Discussion}

Based on the online Algaebase data which includes marine algal species, Guiry and Guiry (2019) provided information about Cystoseira species diversity and discussed the description of new taxa and distribution. In Morocco, some authors have discussed the 
geographic distribution of Bushy Rainbow Wrack in the Mediterranean sea of Morocco (Ribera et al., 1992; Báez et al., 2005; Taskin et al., 2012; Bermejo et al., 2018; Moussa et al., 2018). However, no studies have been made in the Atlantic coast of Morocco concerning these species. Some authors have mentioned their sensitivity (Ballesteros et al., 1984; Thibaut et al., 2005). In contrast, the situation is more critical at Safi coast where only Bushy Rainbow Wrack seems to be more tolerant but all Cystoseira species have disappeared in 3 polluted stations (S2, S4 and S5). Many authors have showed the sensitivity of these brown seaweeds in polluted coasts (Cormaci et al., 1999; Thibout et al., 2005; Sales and Ballesteros, 2009). Furthermore, Cystoseira species are used as indicators of good water quality (Ballesteros et al., 2007).

Table 8. Varimax with Kaiser Normalization rotated loading for 2 components. converged in 3 iterations with Principal Component Analysis extraction method according to heavy metals, physiological parameters in Bushy Rainbow Wrack and physicochemical compounds for the stations studied

\begin{tabular}{|c|c|c|}
\hline \multirow{2}{*}{ Parameter } & \multicolumn{2}{|c|}{ Component } \\
\hline & 1 & 2 \\
\hline $\mathbf{C d}$ & 0.951 & -0.059 \\
\hline $\mathbf{P b}$ & 0.645 & -0.177 \\
\hline $\mathrm{Cu}$ & 0.735 & -0.327 \\
\hline $\mathrm{Cr}$ & 0.961 & -0.097 \\
\hline TP & 0.101 & -0.797 \\
\hline DP & 0.912 & 0.093 \\
\hline $\mathrm{PO}_{4}{ }^{3-}$ & 0.905 & -0.034 \\
\hline TN & -0.113 & 0.569 \\
\hline DN & 0.084 & 0.965 \\
\hline $\mathrm{NO}_{3}{ }^{-}$ & -0.215 & 0.528 \\
\hline $\mathrm{NH}_{4}{ }^{+}$ & -0.653 & 0.162 \\
\hline Chl a & -0.940 & 0.240 \\
\hline Chl c & -0.824 & 0.279 \\
\hline Carotenoid & -0.753 & 0.509 \\
\hline Proline & 0.967 & -0.104 \\
\hline TPC & 0.915 & -0.220 \\
\hline GB & 0.967 & -0.104 \\
\hline Variance (\%) & 57.500 & 16.858 \\
\hline Cumulative (\%) & 57.500 & 74.085 \\
\hline
\end{tabular}

The values of nitrogenous compounds are higher in comparison with the other ocean areas (Sverdrup et al., 1943). It is widely accepted that nitrogen may be an important factor in limiting marine algal productivity. Although the need to examine macroalgalnitrogen relationships has long been recognized (Haas and Hill, 1933). Efforts with benthic marine algae have not paralleled those with phytoplankton.

The highly concentration of phosphorus was due to the huge amount of phosphogypsum used by the industrial factory. Inorganic nutrients are present in natural environments but their concentration near urban areas is usually enhanced (Nixon, 1995; Scavia and Bricker, 2006). The overloading of inorganic nutrients stimulates algal production and increases turbidity leading to changes in the species composition and the structure of littoral communities (Mcglathery et al., 2007). In addition, increased nutrient concentration in seawater favors opportunistic species, while long-lived species such as seagrasses and perennial macroalgae gradually decline (Munda, 1982; Schramm et al., 1999). Thus. the high phosphorus concentration in (S5) could explain the disappearance 
of Cystoseira species. It is known according to Celis-Plá et al. (2014) that nitrate and phosphate represent important macronutrients for macroalgae development and can protect the algae against stress. Moreover, highly concentrations of nutrients in seaweeds can reduce photoinhibition, as it has been observed in Bushy Rainbow Wrack. Other observations showed that nutrient enrichment could also have effects on photosynthesis, photo-protection and biochemical responses (Celis-Plá et al., 2016). Nonetheless, stress biology studies on heavy metal in brown seaweeds has demonstrate different results of molecular, biochemical and physiological effects, as it has been studied in Ascophyllum nodosum (Connan and Stengel, 2011a,b), Fucus vesiculosus (Nielsen and Nielsen, 2010) and Ectocarpus siliculosus (Roncarati et al., 2015; Sáez et al., 2015).

The comparison of our data with those previously studied by some authors (Table 9) shows that the concentrations of heavy metals: $\mathrm{Cd}, \mathrm{Pb}, \mathrm{Cu}$ and $\mathrm{Cr}$ for Bushy Rainbow Wrack in the present study recorded higher values in Cystoseira sp obtained by Akcali and Kucuksezgin (2011) of the Aegean Sea in Turkey, Al-Masri et al. (2003) at the Syrian Coast, Schintu et al. (2010) at Sardinia coast in Italy and Caliceti et al. (2001) at the Venice lagoon in Italy. Nonetheless, lower level of $\mathrm{Pb}$ and $\mathrm{Cu}$ than those reported by Schintu et al. (2010) and Caliceti et al. (2001), respectively.

Table 9. Comparison of heavy metal levels ( $\mu \mathrm{g} / \mathrm{g}$ dry weight) in seaweed Cystoseira sp. from other different locations in the world

\begin{tabular}{|c|c|c|c|c|c|c|}
\hline & Cd & $\mathbf{P b}$ & $\mathbf{C u}$ & $\mathbf{C r}$ & Location & References \\
\hline \multirow{5}{*}{ Cystoseira sp. } & 0.18 & 0.003 & 6.00 & - & Aegean Sea, Turkey & (Akcali and Kucuksezgin, 2011) \\
\hline & 1.72 & 10.3 & 1.80 & _ & Sardinia, Italy & (Schintu et al., 2010) \\
\hline & $0.1-0.5$ & 1.31 & 7.21 & - & Syrian Coast & (Al-Masri et al., 2003) \\
\hline & 0.2 & 5.6 & 21 & 1.5 & Venice lagoon, Italy & (Caliceti et al., 2001) \\
\hline & 2.6 & 4.43 & 10.6 & 2.9 & $\begin{array}{c}\text { Atlantic coast of } \\
\text { Morocco }\end{array}$ & Present study \\
\hline
\end{tabular}

The relatively high levels of Lead in seawater could be attributed to discharges from industries near the study sites that could increase directly this element (leaching from gas stations), as well as leaching at the dump, traffic and leaching of farmlands, can contribute to the highly lead concentration at these stations. However, the lead concentrations at all stations are below the Moroccan standard $500 \mu \mathrm{g} / 1$ (FAO, 2006). For Cadmium detected in polluted waters, concentrations indicate a non-contamination by this element. Indeed, they are all below the limit value of $200 \mu \mathrm{g} / \mathrm{l}$ (FAO, 2006) (Table 10). The concentrations found at these sites, lead us to assume the presence of one or several sources of pollution near the stations above.

Table 10. Comparison of heavy metal levels in seawater ( $\mu \mathrm{g} / \mathrm{l})$ for the studied stations with the Moroccan standards for seawater (FAO, 2006)

\begin{tabular}{c|c|c}
\hline Heavy metals & Sea water & Moroccan norm FAO, 2006 \\
\hline $\mathbf{C d}$ & 1.151 & 200 \\
$\mathbf{P b}$ & 2.563 & 500 \\
$\mathbf{C u}$ & 0.604 & 500 \\
$\mathbf{C r}$ & 0.987 & 2000 \\
\hline
\end{tabular}

The resultant PCA (Fig. 3) presenting the loading of the variables on the two principal components demonstrate that $\mathrm{Cd}, \mathrm{Pb}, \mathrm{Cu}, \mathrm{Cr}, \mathrm{DP}, \mathrm{PO}_{4}{ }^{3-}$, Proline, TPC and GB were the 
dominant correlated positive variables on the PC1 $(0.951 ; 0.645 ; 0.735 ; 0.961 ; 0.912$; 0.905; 0.967; 0.915; 0.967, respectively) while Chla, Chlc and Carotenoid the negatif correlated ones $\left(-0.940 ;-0.824 ;-0.753\right.$, respectively), in addition to $\mathrm{TN}, \mathrm{DN}$ and $\mathrm{NO}_{3}{ }^{-}$, the dominant variables on the PC2 $(0.569 ; 0.965 ; 0.528$, respectively). Indeed, according to PCA results, an important positive correlation and proportionate augmentation in proline, GB and TPC contents was recorded with increase in concentration of heavy metals and excessive Phosphorus in polluted stations mentioned, in addition to a negative correlation with pigments. It is proved by Alia et al. (1999) that Cadmium is the strongest inducer for proline and GB accumulation. Moreover, TPC can be released in stressful conditions from algal thalli and could react rapidly with carbohydrates and proteins to form UV-absorbing exudates (Koivikko et al., 2005). It has also been shown that proline, GB and TPC are the stress-induced substances in plants and algae under different kind of stress (Anbazhangan et al., 1988; Fadma et al., 2007; Abdel Latef and Sallam, 2015). Proline and GB concentrations in Bushy Rainbow Wrack in polluted areas are much higher than those found in other green, brown and red seaweeds (Fleurence, 2004). Furthermore, the maximum value of TPC in this study $(1.54 \mathrm{mg} / \mathrm{g}$ dry weight during autumn season at (S8) The port station in Essaouira city) is higher than that found by Pereira and Yoneshigue (1999) in brown seaweed Sargassum furcatum (0.2-0.5 mg/g DW). According to this author, TPC may also be present in brown seaweed for reasons other than defense, as they have been considered to have a number of physiological and ecological functions.

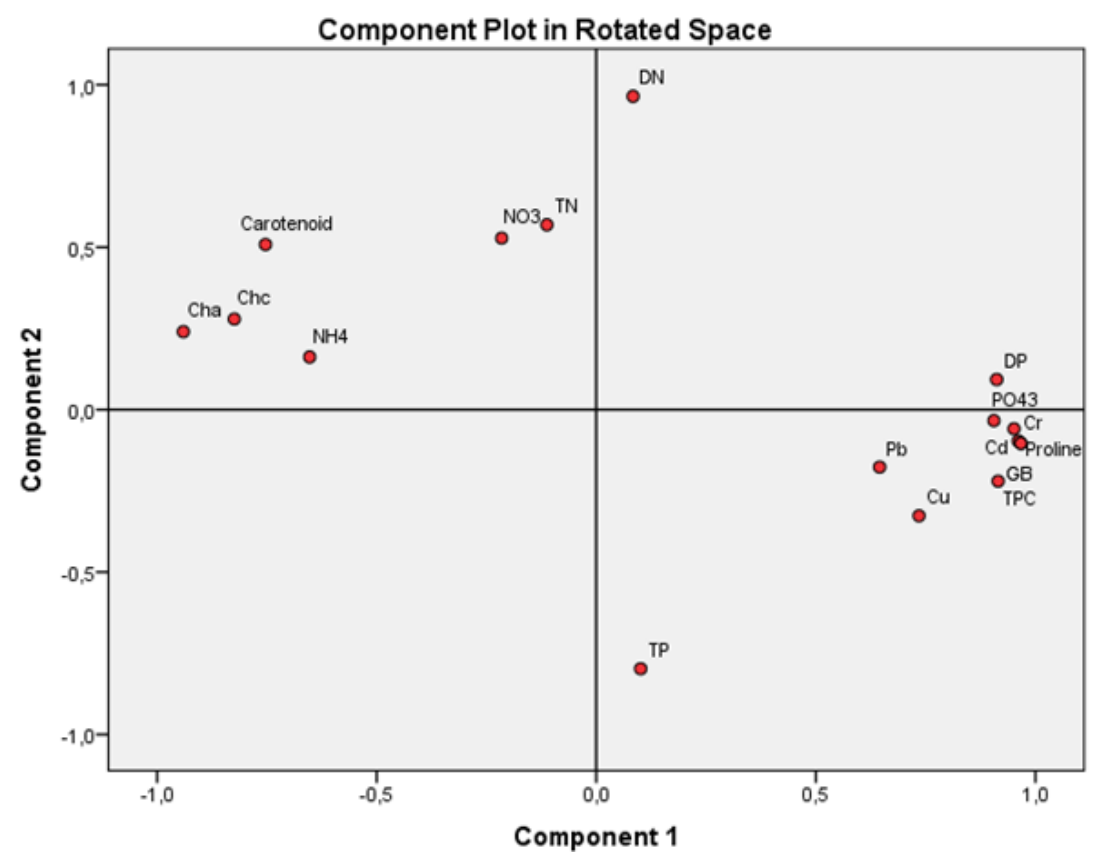

Figure 3. Component Plot in Rotated Space for the parameters studied

In the basis of the Conceptual framework of Mineur et al. (2014), anthropogenic local stressors create additional disruption often altering dramatically assemblage's structure. Global stressors are not manageable locally, but have local impacts and may indirectly affect local stressors. All stressors are affecting seaweed diversity (populations and communities), and directly impacting coastal ecosystems (Fig. 4) (Mineur et al., 2014). 
At the Industrial area (S4) and Phosphate area (S5) in Safi city and Jorf Lasfar area (S2) near Eljadida city, these stressors are due to increased nutrient inputs, heavy metal pollution and mostly derived from the industrial activity out there. According to Ferreira et al. (2011), marine biota living in coastal waters are under constant threat from exposure to elevated concentrations of pollutants, such as metals and nutrients, mostly derived from domestic, industrial and farming activities.

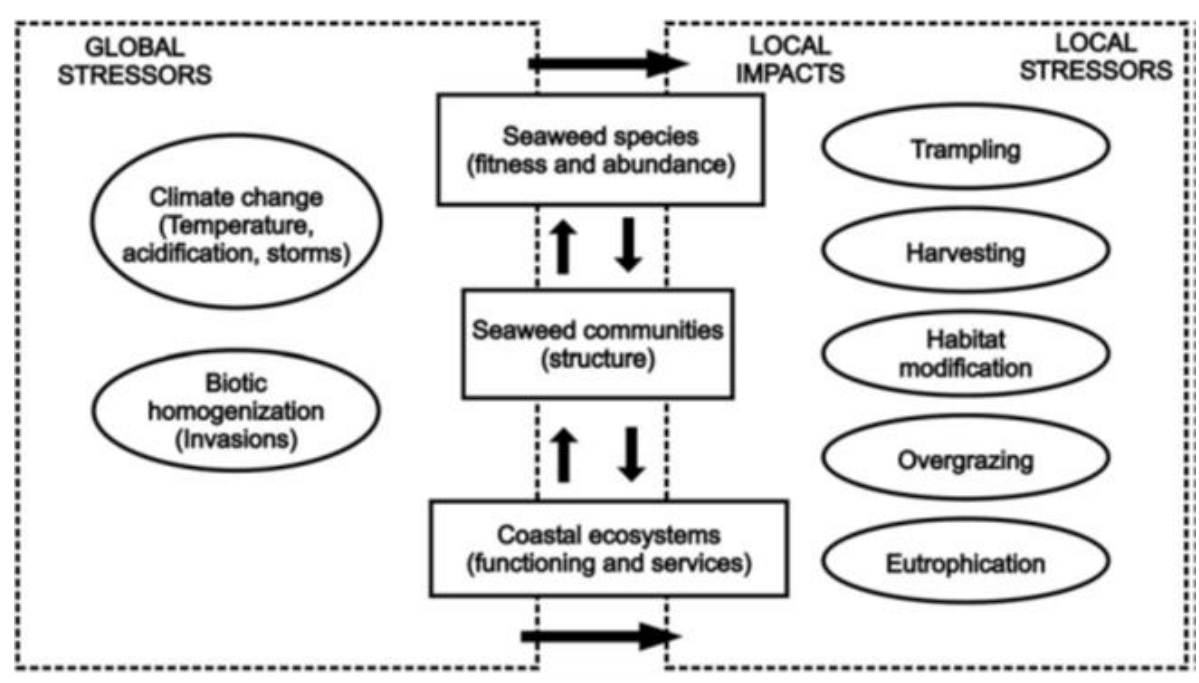

Figure 4. Conceptual framework of Mineur et al. (2014)

Changes in the composition of Bushy Rainbow Wrack through these stressors in polluted stations (S2, S4 and S5) has resonated through entire coastal ecosystems in this area. Furthermore, anthropogenic activities on these coastal areas, such as industrialization and urbanization create increasing anthropogenic stress. Cystoseira and seaweed biodiversity in general of the Moroccan coastlines need more attention to predict how they are affected by human activities.

\section{Conclusion}

To conclude, we have found a regular element variety in species lavishness with least qualities in winter and an auxiliary least top in spring, and most extreme qualities in summer. The regularity is for the most part because of the adjustment in biomass of the overwhelming species. Just Bushy Rainbow Wrack is by all accounts progressively tolerant to the contamination in polluted stations. Nitrogenous compounds did not clarify the decay of the species around there, yet the highly phosphorus concentration in the phosphate station and highly values of heavy metal concentrations could affect the physiology and clarify the vanishing of Bushy Rainbow Wrack, notwithstanding the nearness of dangerous components like $\mathrm{Pb}, \mathrm{Cd}, \mathrm{Cu}$ and $\mathrm{Cr}$.

Thus, the investigation on the combined effects of nutrients, metals and biochemical parameters studied in this brown seaweed would provide relevant information about their capacity to withstand the future pollution scenarios. The interaction between metals and nutrients excess is still not well understood for macroalgae. Further examinations ought to be done to characterize different parameters that lead to Bushy Rainbow Wrack debasement. 
For future research, we aim to develop the culture experiments under controlled laboratory conditions on Bushy Rainbow Wrack as well as the genetic characterization of this brown seaweed based upon DNA barcoding of the cytochrome oxidase subunit 1 (COI), 23S rDNA (23S), and 23S-tRNAVal intergenic spacer (mt-spacer).

\section{REFERENCES}

[1] Abdel Latef, A. A., Sallam, M. M. (2015): Changes in Growth and Some Biochemical Parameters of Maize Plants Irrigated with Sewage Water. - Austin Journal of Plant Biology 1: 1004.

[2] AFNOR NF T90-012. Août (1975): Dosage des nitrates.

[3] AFNOR NF T90-015-2. Janvier (2000): Qualité de l'eau. Dosage de l'ammonium. - Partie 2: méthode spectrométrique au bleu d'indophénol.

[4] AFNOR NF T90-022. Avril (2005): Dosage des orthophosphates.

[5] Akcali, I., Kucuksezgin, F. (2011): A biomonitoring study: heavy metals in macroalgae from eastern Aegean coastal areas. - Marine Pollution Bulletin 62: 637-645.

[6] Alia, F., Saradhi, P. P. A. (1991): Proline Accumulation Under Heavy Metal Stress. - Plant Physiology 138: 554-558.

[7] Al-Masri, M. S., Mamish, S., Budier, Y. (2003): Radionuclides and trace metals in eastern Mediterranean Sea algae. - Journal of Environmental Radioactivity 67: 157-168.

[8] Anbazhagan, M., Krishnamurthy, R., Bhagwat, K. A. (1988): Proline: an enigmatic indicator of air pollution tolerance in rice cultivars. - Journal of Plant Physiology 133: 122123.

[9] Báez, J. C., Olivero, J., Real, R., Vargas, J. M., Flores-Moya, A. (2005): Analysis of geographical variation in species richness within the genera Audouinella (Rhodophyta). Cystoseira (Phaeophyceae) and Cladophora (Chlorophyta) in the western Mediterranean Sea. - Botanica Marina 48: 30-37.

[10] Ballesteros, E., Perez, M., Zabala, M. (1984): Aproximacion al conocimiento de las comunidades algales de la zona infralitoral superior en la costa catalana. - Collectane A Botanica 15: 69-100.

[11] Ballesteros, E., Torras, X., Pinedo, S., Garcia, M., Mangialajo, L., De Torres, M. (2007): A new methodology based on littoral community cartography for the implementation of the European Water Framework Directive. - Marine Pollution Bulletin 55: 172-180.

[12] Bermejo, R., Chefaoui, R. M., Engelen, A. H., Buonomo, R., Neiva, J., Ferreira-Costa, J., Pearson, G. A., Marbà, N., Duarte, C. M., Airoldi, L., Hernández, I., Guiry, M. D., Serrão, E. A. (2018): Marine forests of the Mediterranean-Atlantic Cystoseira tamariscifolia complex show a southern Iberian genetic hotspot and no reproductive isolation in parapatry. - Science Reports 8: 1-13.

[13] Blinda, M., Bellaouchou, A., Fekhaoui, M., Barcha, S.-E., El Morhit, M. (2013): Assessment of metal contamination of the sediments and superficial waters in the northwest coast of Morocco. - Bulletin de l'Institut Scientifique 35: 43-49.

[14] Caliceti, M., Argese, E., Sfriso, A., Pavoni, B. (2001): Heavy metal contamination in the seaweeds of the Venice lagoon. - Chemosphere 47: 443-454.

[15] Celis-Plá, P. S. M., Bouzon, Z. L., Hall-Spencer, J. M., Schmidt, E. C., Korbee, N., Figueroa, F. L. (2016): Seasonal biochemical and photophysiological responses in the intertidal macroalga Cystoseira tamariscifolia (Ochrophyta). - Marine Environmental Research 115: 89-97.

[16] Celis-Plá, P. S. M., Martínez, B., Quintano, E., García-Sánchez, M., Pedersen, A., Navarro, N. P., Copertino, M. S., Mangaiyarkarasi, N., Mariath, R., Figueroa, F. L., Korbee, N. (2014): Short-term ecophysiological and biochemical responses of Cystoseira 
tamariscifolia and Ellisolandia elongata to environmental changes. - Aquatic Biology 22: 227-243.

[17] Cherifi, O., Sabri, H., Gharmali, A., Maarouf, A., Hasni, M., Cherifi, K., Ait-Hman, A., Derhem, A., Bahammou, N., Sbihi, K. (2018): Variation spatio-temporelle des métaux traces $(\mathrm{Cr}, \mathrm{Cu}, \mathrm{Pb}, \mathrm{Zn})$ chez la laminariale Saccorhiza polyschides au niveau du littoral de la région d'Essaouira. - SMETox Journal 1: 53-58.

[18] Claudet, J., Fraschetti, S. (2010): Human-driven impacts on marine habitats: a regional meta-analysis in the Mediterranean Sea. - Biological Conservation 143: 2195-2206.

[19] Connan, S., Stengel, D. B. (2011a): Impacts of ambient salinity and copper on brown algae: 2. Interactive effects on phenol pool and assessment of metal binding capacity of phlorotannin. - Aquatic Toxicology 104: 1-13.

[20] Connan, S., Stengel, D. B. (2011b): Impacts of ambient salinity and copper on brown algae: 1. Interactive effects on photosynthesis. growth. and copper accumulation. - Aquatic Toxicology 104: 94-107.

[21] Cormaci, M., Furnari, G. (1999): Changes of the benthic algal flora of the Tremiti Islands (southern Adriatic) Italy. - In: Kain, J. M., Brown, M. T., Lahaye, M. (eds.) Sixteenth International Seaweed Symposium. Developments in Hydrobiology. Springer. Dordrecht.

[22] Dayton, P. K. (1975): Experimental evaluation of ecological dominance in a rocky intertidal algal community. - Ecological Monographs 45: 137-159.

[23] Essedaoui, A., Sif, J. (2001): Bioaccumulation des métaux lourds et induction des métalloprotéines au niveau de la glande digestive de Mytilus galloprovincialis. - Revue Marocaine des Sciences Agronomiques et Vétérinaires 21: 17-25.

[24] F.M.6. (2018): The Mohammed VI Foundation for the Protection of the Environment. Annual report, Morocco.

[25] FAO. (2006): Projet de gestion des ressources en eau : Elaboration des dossiers techniques relatifs aux valeurs limites des rejets industriels dans le Domaine Public Hydraulique. Elaboration des fiches techniques des valeurs limites des rejets industriels. - Convention FAO/UTF/MOR019/MOR.

[26] Fatma, T., Khan, M. A., Choudhary, M. (2007): Impact of environmental pollution on cyanobacterial proline content. - Journal of Applied Phycology 19: 625-629.

[27] Ferreira, J. G., Andersen, J. H., Borja, A., Bricker, S. B., Camp, J., Cardoso da Silva, M., Garcés, E., Heiskanen, A. Z., Humborg, C., Ignatiades, L., Lancelot, C., Menesguen, A., Tett, P., Hoepffner, N., Claussen, U. (2011): Overview of eutrophication indicators to assess environmental status within the European Marine Strategy Framework Directive. Estuary Coastal Shelf Science 93: 117-131.

[28] Ferssiwi, A., Sif, J., El Hamri, H., Rouhi, A., Amiard, J. C. (2004): Contamination par le cadmium de l'Annélide Polychète Hediste diversicolor dans la région d'El Jadida (Maroc) implication des protéines type métallothionéines. - Journal de recherche océanographique 29: 59-64.

[29] Fleurence, J. (2004): Seaweed proteins. - University of Nantes, France.

[30] Goumri, M., Cheggour, M., Maarouf, A., Mouabad, A. (2018): Preliminary data on the composition and spatial distribution patterns of echinoderms along Safi rocky shores (NW Morocco). - AACL Bioflux 11(4): 1193-1202.

[31] Grieve, C. M., Grattan, S. R. (1986): Rapid assay for determination of water soluble quaternary ammonium compounds. - Plant and Soil 70: 303-307.

[32] Guiry, M. D, Guiry, G. M. (2019): AlgaeBase. - World-wide electronic publication, National University of Ireland, Galway.

[33] Haas, P., Hill, T. G. (1933): Observations on the metabolism of certain seaweeds. - Annals of Botany 47: 55-67.

[34] Jeffrey, S. W., Humphry, G. F. (1975): New Spectrophotometric Equations for Determining Chlorophylls a, b, c1 and c2 in Higher Plants, Algae and Natural Phytoplankton. - Biochimie und Physiologie der Pflanzen (BPP) 167: 191-194.

[35] Jones, A. (1997): Environmental biology. - Routledge, London. 
[36] Kaimoussi, A., Chafik, A., Mouzdahir, A., Bakkas, S. (2001): The impact of industrial pollution on the Jorf Lasfar coastal zone (Morocco, Atlantic Ocean): the mussel as an indicator of metal contamination. - Comptes Rendus, Academie des Sciences, Paris 333: 337-341.

[37] Koivikko, R., Loponen, J., Honkanen, T., Jormalainen, V. (2005): Contents of soluble cellwall-bound and exuded phlorotannins in the brown alga Fucus vesiculosus with implications on their ecological functions. - Journal of Chemical Ecology 31(1): 195-212.

[38] Lichtenthaler, H. K. (1987): Chllorphyls and carotenoids: Pigments of photosynthetic biomembranes. - Methods in Enzymology 148: 350-380.

[39] Mcglathery, K. J., Sundbäk, K., Anderson, I. C. (2007): Eutrophication in shallow coastal bays and lagoons: the role of plants in the coastal filter. - Marine Ecology Progress Series 348: 1-18.

[40] Mineur, F., Arenas, F., Assis, J., Davies, A. J., Engelen, A. H., Fernandes, F., Malta, E. J., Thibaut, T., Nguyen, T. V., Vaz-Pinto, F., Vranken, S., Serrão, E. A., De Clerck, O. (2014): European seaweeds under pressure: Consequences for communities and ecosystem functioning. - Journal of Sea Research 98: 91-108.

[41] Monneveux, P., Nemmar, M. (1986): Contribution à l'étude de la résistance à la sécheresse chez le blé tendre (Triticum aestivum L.) et chez le blé dur (Triticum durum Desf.): étude de l'accumulation de la proline au cours du cycle de développement. - Agronomie 6: 583590.

[42] Moussa, H., Hassoun, M., Salhi, G., Zbakh, H., Riadi, H. (2018): Checklist of seaweeds of Al-Hoceima National Park of Morocco (Mediterranean Marine Protected Area). - Acta Botanica Malacitana 43: 91-109.

[43] Munda, I. M. (1982): The effects of organic pollution on the distribution of fucoid algae from the Istrian coast (vicinity of Rovinj). - Acta Adriatica 23: 329-337.

[44] Munday, P. L., Warner, R. R., Monro, K., Pandolfi, J. M., Marshall, D. J. (2013): Predicting evolutionary responses to climate change in the sea. - Ecology Letters 16: 1488-1500.

[45] NF EN ISO 11905-1. Juillet (1998): Qualité de l'eau. Dosage de l'azote. - Partie 1: Méthode par minéralisation oxydante au peroxodisulfate (indice de classement T 90-061).

[46] NF EN ISO 6878. Avril (2005): Qualité de l'eau. Dosage du phosphore: méthode spectrophotométrique au molybdate d'ammonium (indice de classement T90-023).

[47] Nielsen, H. D., Nielsen, S. L. (2010): Adaptation to high light irradiances enhances the photosynthetic $\mathrm{Cu}^{2+}$ resistance in $\mathrm{Cu}^{2+}$ tolerant and non-tolerant populations of the brown macroalgae Fucus serratus. - Marine Pollution Bulletin 60: 710-717.

[48] Nixon, S. W. (1995): Coastal marine eutrophication: a definition, social causes and future concerns. - Ophelia 41: 199-219.

[49] Pereiraa, R. C., Yoneshigue, V. Y. (1999): The Role of Polyphenols from the Tropical Brown Alga Sargassum furcatum on the Feeding by Amphipod Herbivores. - Botanica Marina 42: 441-448.

[50] Ribera, M. A., Gómez-Garreta, A., Gallardo, T., Cormaci, M., Furnari, G., Giaccone, G. (1992): Check-list of Mediterranean Seaweeds. I. Fucophyceae (Warming 1884). Botanica Marina 35: 109-130.

[51] Roncarati, F., Sáez, C. A., Greco, M., Gledhill, M., Bitonti, M. B., Brown, M. T. (2015): Response differences between Ectocarpus siliculosus populations to copper stress involve cellular exclusion and induction of the phytochelatin biosynthetic pathway. - Aquatic Toxicology 159: 167-175.

[52] Sabri, H., Cherifi, O., Maarouf, A., Cheggour, M., Bertrand, M., Mandi, L. (2017): Wastewater impact on macroalgae biodiversity in Essaouira coast (Morocco). - Journal of Materials and Environmental Sciences 8: 857-862.

[53] Sáez, C. A., Roncarati, F., Moenne, A., Moody, A. J., Brown, M. T. (2015): Copperinduced intra-specific oxidative damage and antioxidant response of the brown alga Ectocarpus siliculosus with different pollution histories. - Aquatic Toxicology 159: 81-89. 
[54] Sales, M., Ballesteros, E. (2009): Shallow Cystoseira (Fucales: Ochrophyta) assemblages thriving in sheltered areas from Menorca (NW Mediterranean): relationships with environmental factors and anthropogenic pressures. - Estuarine Coastal and Shelf Science 84: 476-482.

[55] Scavia, D., Bricker, S. B. (2006): Coastal eutrophication assessment in the United States. - Biogeochemistry 79: 187-208.

[56] Schintu, M., Marras, B., Durante, L., Meloni, P., Contu, A. (2010): Macroalgae and DGT as indicators of available trace metals in marine coastal waters near a lead-zinc smelter. Environmental Monitoring and Assessment 167: 653-661.

[57] Schramm, W. (1999): Factors influencing seaweed responses to eutrophication: some results from EU-project EUMAC. - In: Kain, J. M., Brown, M. T., Lahaye, M. (eds.) Sixteenth International Seaweed Symposium. Developments in Hydrobiology. Springer. Dordrecht.

[58] Sverdrup, H. U., Johnson, M. W., Fleming, R. H. (1943): The Oceans, their physics, chemistry, and general biology. - Physiological Zoology 16: 322-323.

[59] Taga, S. M., Miller, E. E., Pratt, D. E. (1984): Chia seeds as a source of natural lipid antioxidants. - JAOCS 61: 928.

[60] Taskin, E., Jahn, R., Öztürk, M., Furnari, G., Cormaci, M. (2012): The Mediterranean Cystoseira (with photographs). - Photographs. Celar Bayar University. Manisa. Turkey.

[61] Thibaut, T., Pinedo, S., Torras, X., Ballesteros, E. (2005): Long-term decline of the populations of Fucales (Cystoseira spp. and Sargassum spp.) in the Albères coast (France north-western Mediterranean). - Marine Pollution Bulletin 50: 1472-1489.

[62] Topcuoglu, S., Güven, K.C., Balkis, N., Kibasoglu, C,., 2003. Heavy metal monitoring of marine algae from the Turkish Coast of the Black Sea, 1998-2000. - Chemosphere 52: $1683-1688$.

[63] Walker, D. I., Kendrick, G. A. (1998): Threats to macroalgal diversity: Marine habitat destruction and fragmentation, pollution and introduced species. - Botanica Marina 41: 105-112. 